\section{P.2.38 OCCUPATIONAL EXPOSURE AND INCIDENCE OF ASTHMA BASED ON PRESCRIPTION OF DRUGS}

\begin{abstract}
${ }^{1,2}$ Jesper Bælum, ${ }^{3}$ Lars Rauff Skadhauge, ${ }^{4}$ Trine Thilsing, ${ }^{5}$ Jesper Rønhild Davidsen, ${ }^{6}$ Ojvind Omland, ${ }^{7}$ Vivi Schlünssen*. 'Dept. of Occupational and Environmental Medicine, Odense University Hospital, Odense, Denmark; ${ }^{2}$ OPEN, Odense Patient data Explorative Network, Odense University Hospital, Odense, Denmark; ${ }^{3}$ Dept. of Occupational Medicine, Hospital of South West Jutland, Esbjerg, Denmark; ${ }^{4}$ Dept. of Public Health, University of Southern Denmark, Odense, Denmark; ${ }^{5}$ Dept. of Respiratory Medicine, Odense University Hospital, Odense, Denmark; ${ }^{6}$ Dept. of Occupational Medicine, Aalborg University Hospital, Alborg, Denmark; ${ }^{7}$ Dept. of Public Health, Aarhus University, Aarhus, Denmark
\end{abstract}

\subsection{6/OEM-2019-EPI.265}

Prescription of drugs for obstructive lung diseases (ATC code R03) has previously been shown to be an indicator of actual asthma. In this cohort study, we have combined occupational information with data from redeemed prescriptions between 2000 and 2013 extracted from the National Danish Prescription register.

In 2003 a total of 7255 persons aged 20 and 44 years fulfilled a questionnaire, which among other things, included information on their longest held job. The jobs of 6470 were coded according to ISCO-88 and an asthma Job Exposure Matrix (JEM) was applied. Prevalent asthma was defined as at least two redeemed prescriptions of a R03 drug within 2 years. Incident asthma between 2003 and 2013 was defined as not having redeemed a R03 prescription in the previous years. Data was analyzed separately for each gender using multivariate logistic regression and presented as odds ratios (OR) with 95\% confidence intervals (CI).

Among those having a job 327 (5.1\%) were identified as incident cases and $467(7.2 \%)$ as prevalent cases. In females increased incidences were seen in exposures to reactive low molecular weight (LMW) substances (OR1.47 (95\% CI 1.042.07)), cleaning agents (OR 1.52 (1.05-2.18)), metals (OR 3.31 (1.63-6.64)), while increased prevalence was seen with mite exposure (OR $4.41(1.74-11.2)$ ) and irritant gases (OR 1.76 (1.16-2.69)). In males no increased incidences were seen and only an increased prevalence with mixed environments (OR 2.24 (1.13-4.43)).In jobs increased prevalence and incidence were seen in female cleaners and drivers. Increased prevalence was seen in male printing workers.

Meaningful associations with well-known asthmagenic exposures in young adults with asthma can be identified in administrative register data, and implementing the analyses of register data from larger populations will have the power to detect potential increased risks due to rare exposures or changes in risk over time.

\section{P.2.39 PERICONCEPTIONAL EXPOSURE TO AMBIENT AIR POLLUTION AND CONGENITAL HYPOSPADIAS}

${ }^{1}$ Ching-chun Huang* ${ }^{*}, 2 Y$ ue Leon Guo. ${ }^{1}$ Department of Environmental and Occupational Medicine, National Taiwan University College of Medicine and Hospital, Taipei, Taiwan; ${ }^{2}$ National Institute of Environmental Health Sciences, National Health Research Institutes, Miaoli, Taiwan

\subsection{6/OEM-2019-EPI.266}

Background Evidence regarding whether prenatal exposure to air pollution increases the risk of hypospadias is limited.

Objectives The aim of the study is to evaluate the association between exposure to ambient air pollution during early pregnancy and occurrence of hypospadias.
Methods We conducted a 1:10 case-control study using the Taiwanese Birth Registry database. Those male births reported to have hypospadias were defined as cases; while controls were randomly, matched by birth year, selected from those male births without any congenital anomaly. Monthly average of ambient air pollutants, including PM10, PM2.5, NO2, $\mathrm{NOx}$, and $\mathrm{O} 3$, from three months pre- to six months postconception were retrieved from the 76 air quality monitoring stations and interpolated to the level of township using empirical bayesian kriging. Potential covariates to be adjusted included gestational age, birth weight, birth season, maternal age, maternal diabetes and hypertension, maternal smoking, annual household income and population density of the residential township.

Results During 2007-2014, a total of 265 hypospadias was reported, and 230 (87\%) of them were full-term births. Results of multivariate logistic regression models revealed that for per IQR increase of O3 (8.0 p.p.b) exposure during the first months after conception increased the risk of hypospadias $(\mathrm{aOR}=1.38,95 \% \mathrm{CI}=1.07-1.78)$. In subgroup analysis of fullterm births, we further found that PM2.5 exposure during the first three months post-conception significantly increased the risk of developing hypospadias $(\mathrm{aOR}=1.29$, 95\% $\mathrm{CI}=1.01$ 1.65 , per $\mathrm{IQR}=15.4 \mathrm{ug} / \mathrm{m} 3)$.

Conclusions The results of the study suggested that early gestational exposure to ambient air pollution increased the risk of hypospadias occurrence.

\section{P.3.03 LOW BACK PAIN AND MANUAL PATIENT HANDLING AMONG HEALTHCARE WORKERS: A CROSS-SECTIONAL STUDY}

${ }^{1}$ Adriano Dias*, ${ }^{1}$ João Marcos Bernardes. 'Unesp - São Paulo State University, Botucatu Medical School, Botucatu, Brazil

\subsection{6/OEM-2019-EPI.267}

This cross-sectional study investigated the prevalence of low back pain and analyzed whether manual patient handling activities (MPH) were related to low back pain (LBP) in healthcare workers (HCW). Participants were HCWs working at the University Hospital of Botucatu Medical School, who were willing to participate and provided written informed consent. Of the $488 \mathrm{HCWs}$ who were invited, 320 participated for a response rate of $65,6 \%$. Data were collected using a self-administered anonymous paper questionnaire containing seven parts: manual patient handling, low back pain, demographic characteristics, occupational variables, psychosocial work conditions, lifestyle variables and history of low back pain. The association between MPH and LBP was analyzed using a Poisson regression model. Initially, simple Poisson regression models with robust variance were conducted for each covariate. Those variables with a $p$ value $\leq 0.25$ were selected for inclusion in a multiple Poisson regression model with robust variance, and those with $\mathrm{p}<0.05$ were considered statistically significant. Most respondents were female (83.8\%), the mean age was 39.93 years (range 21 - 66, SD 9.78). Of those participating in the study, the majority were nursing technicians (71.3\%), 19.4\% were nurses, 5.3\% were nurse assistants, $2.2 \%$ were physical therapists and $1.9 \%$ were radiography technicians. The 3 -month period prevalence of LBP was 57.8\% (95\% CI 52.3\%-63.2\%). In the multiple Poisson regression model, MPH (PR 1.444, 95\% CI 1.169-1.783) 